\title{
ESCUELA MEDIA Y ABANDONO ESCOLAR EN ARGENTINA: APROXIMACIONES A UN DEBATE PENDIENTE
}

RESUMEN: Este texto tiene como objetivo exponer panorámicamente un recuento del surgimiento histórico de la educación media en Argentina, para avanzar luego en la evolución de la expansión matricular hasta la actualidad, contemplando los rasgos fundamentales de la misma. A partir de allí, se expondrán factores incidentes en la problemática del abandono escolar, prestando énfasis a las características de la modalidad técnica. Se retomarán puntos centrales de un debate escasamente explorado que es aquel que aborda la intersección específica entre abandono escolar y los estudiantes de modalidad técnica. Se subraya que las condiciones del mercado laboral actual, como la amplitud de la precarización, el avance del sector servicios y la estrechez en materia de industrialización tornan aún más acuciante el contorno de discusiones sobre esta problemática. Este panorama marcha en paralelo a cambios de envergadura en el sistema educativo nacional, enmarcados en sucesivas modificaciones a las normativas jurídicas que lo orientan.

Palabras claves: Escuela Media. Abandono Escolar. Modalidad Técnica.

\section{MIDDLE SCHOOL AND DROPOUT IN ARGENTINA: APPROACHES TO AN UNRESOLVED DEBATE}

ABSTRACT: This text aims to expose panoramically a recount of the historical emergence of secondary education in Argentina, to advance later in the evolution of the expansion currently watching enrolling until the fundamental features of it. From there, it will expose the factors which affect the dropout problem giving particular emphasis to the characteristics of the technical arrangement. Focal points will resume in a little explored that debate is one that addresses the specific intersection between ESL students and technical arrangement. It emphasizes that current labor market conditions, as the amplitude of the precarious, the advance of the service sector and the narrowness in industrialization becomes even more pressing the outline of discussions on this issue. Panorama marching in parallel with major changes in the national education system, surrounded by successive amendments to legal regulations that guide the system.

Keywords: Middle School; ESL; Technical Arrangement.

\footnotetext{
* Doctora en Ciencias Sociales de la Facultad Latinoamericana de Ciencias Sociales (FLACSO). Investigadora Asistente CONICET; Investigadora Programa sobre Juventud de la Facultad Latinoamericana de Ciencias Sociales (FLACSO), Sede Académica Argentina. E-mail: aotero14@gmail.com
} 


\section{Introducción}

Buena parte de la literatura y de las investigaciones del ámbito local, desde fines del siglo XX hasta la actualidad, han puesto el acento sobre distintos aspectos de las transformaciones producidas en la estructura social de la Argentina y en la fisonomía de la sociedad. Durante décadas el país fue considerado una sociedad "relativamente integrada" en un contexto de tendencia hacia el pleno empleo, dotada de un conjunto de instituciones que, ligadas a esta condición, posibilitaron la incorporación de un amplio sector de los trabajadores en términos de derechos, protecciones sociales y estabilidad laboral.

En efecto, al promediar el siglo XX, la primacía del modelo industrialista y la expansión del empleo como forma típica de vinculación al mercado laboral, fueron algunos de los factores promotores de esta idea de integración social basada en la inserción vía mercado de trabajo1. Asimismo, se destacaba "el desarrollo temprano del sistema educativo argentino"; un sistema escolar diseñado en estrecha relación con la constitución del Estado - Nación a partir del XIX, cuya creación contribuyó a la conformación de una amplia clase media. Esta pintura, a su vez, vertebró una sociedad con pautas menos clasistas, menos segmentadas y más integradas que otras sociedades de la región (TEDESCO, 1982; TIRAMONTI, 2004) $)^{2}$.

Sin embargo, la imagen de un "país con cierta capacidad inclusiva" comenzó a diluirse tras la percepción de crisis y mutación de la identidad social. Aquella promesa de una sociedad más integrada y homogénea, dentro del concierto de los países de la región de América Latina, se fue desvaneciendo hacia fines del siglo XX. Paralelamente se daba la pérdida de la capacidad regulatoria de la institución estatal en tanto vértice de la pirámide social, lo cual subyacía en la evolución del proceso de fragmentación social al tiempo que avanzaban la desigualdad y la exclusión social. Debacle que aparece principalmente asociada al peso de las problemáticas vinculadas al mundo laboral. ${ }^{3}$

Los procesos de fragmentación y atomización social han ido en incremento y si bien la dinámica de transformaciones dista de ser reciente, los inicios de la década del 90 significaron la aplicación de medidas de ajuste estructural como la apertura del mercado, la privatización de las empresas públicas y la flexibilización de los contratos de trabajo, cuyos 
impactos contribuyeron al deterioro de las condiciones de vida de gran parte de la población. Esta situación quedó expresada a través de indicadores como niveles inéditos de desempleo, desigualdad distributiva y pobreza. Con la sanción de la Ley Nacional de Empleo, 24.013, en 1991, el gobierno nacional materializó una transformación significativa para dar curso a la flexibilización del mercado de trabajo. La ley estableció distintas modalidades de contratación laboral promovidas mediante rebajas o eliminación de cargas sociales y definió cambios en los métodos de ajuste salarial, promoviendo cláusulas del tipo "ajuste a la productividad". Tales medidas, finalmente, contribuyeron a la degradación de las condiciones laborales del conjunto de los trabajadores (MARSHALL, 1998). ${ }^{4}$

\section{Sistema educativo argentino y evolución matricular}

Históricamente, el sistema escolar argentino tiene su raigambre a fines del 1800 y estuvo fuertemente vinculado a la proyección de una escuela absolutamente imbricada con los orígenes del Estado. En el terreno de la educación cabe precisar que el proyecto modernizador desplegado sentaba las bases para la integración a un orden social por medio de la socialización de las nuevas generaciones a partir de la escuela. El desarrollo de dicho sistema, como mencionáramos, tuvo una presencia medular en la constitución de la Nación y su sentido social se definió asociado a la formación ciudadana.

El mismo contó con una temprana y dinámica expansión a lo largo de todo el territorio, pero con considerables variables regionales. En cuanto a la evolución histórica del tramo de educación secundaria / nivel medio, este parte de mediados del siglo XIX. Se ha advertido sobre el carácter selectivo y elitista de su origen, dado su intrincada vinculación con los procesos de reproducción ampliada de las elites en un contexto de crecimiento de la sociedad industrial y urbana que acompasaba el proceso de incorporación de la economía argentina en el sistema capitalista mundial. En aquel entonces, esta experiencia de aprendizaje era accesible para pocos privilegiados. Para algunos, el exitoso pasaje por la secundaria era puerta de entrada a la universidad; para otros, habilitaba puestos de trabajo formales en instituciones públicas y privadas, sobre todo en las capitales provinciales del país (TENTI FANFANI, 2003). 
La primera escuela secundaria, el "Colegio Nacional de Buenos Aires", fue fundada hacia el año 1863, en el marco de una política educativa que multiplicó la experiencia, creando en las principales capitales provinciales otros colegios nacionales destinados a la formación política y la continuidad educativa universitaria de las elites. En paralelo, hacia 1965, se inició la expansión de las escuelas normales tras el objetivo primario de la formación de maestros que pudieran sostener la notable expansión del nivel primario de enseñanza (DUSSEL, 1997).

No obstante, las escuelas normales no se definieron como una oferta de educación secundaria en sus inicios, tal como fueron reconocidas posteriormente durante el siglo XX. En el año 1969 la formación docente se redefinió, convirtiéndose en una oferta de nivel superior nouniversitario. Y, a partir de 1970, las escuelas normales acabaron incluidas a la modalidad denominada bachiller.

Por su parte, la modalidad técnica tuvo sus inicios hacia el año 1871, en departamentos anexos de los colegios nacionales de tres provincias: Salta, San Juan y Catamarca. En este sentido, las orientaciones técnicas, productivas y comerciales se fundaron en el marco de los colegios nacionales, como anexos de los mismos. Posteriormente, se creó el departamento industrial de la Escuela de Comercio de Buenos Aires, el cual se independizó hacia 1899, transformándose en el primer colegio industrial de la Argentina, actualmente "Otto Krause". Otro antecedente histórico de peso ha sido la Escuela de Comercio de la Capital de la República, hoy "Carlos Pellegrini", creada hacia 1890, cuyos objetivos centrales incluían la formación en matemática, el cálculo mercantil, el manejo de libros contables y los idiomas extranjeros. (MIRANDA; OTERO; CORICA, 2008).

En cuanto a su performance, diversos especialistas han apuntado que la educación técnica fue la modalidad que experimentó mayores transformaciones durante el siglo XX. Alcanzó su mayor desarrollo a partir de 1946, cuando se creó la Comisión Nacional de Orientación y Aprendizaje. Luego, hacia el año 1959, sus distintas versiones fueron agrupadas en torno al Consejo Nacional de Educación Técnica (CONET) y, a partir de 1965, adquirieron la denominación de Escuelas Nacionales de Educación Técnica (ENETs). Esta última estructura ha sido la que permaneció hasta la década del 90.

La evolución de la matrícula escolar del nivel medio ha ido en incremento registrando los mayores porcentajes a lo largo de la segunda 
mitad del siglo XX, no obstante, en buena parte del periodo, el incremento estuvo relacionado con la expansión de la modalidad técnica. La notable ampliación matricular técnica se registra desde los años 40 en directa relación con el avance del modelo industrialista, auspiciando procesos de movilidad social ascendente. En cierta forma, la educación secundaria comenzaba así a declinar su mandato original de constituirse como un privilegio de las elites, para convertirse en el principal canal que brindaba sustento a la movilidad social de nuevas clases medias.

Notoriamente, desde los años 40 hasta mediados de la década del 70 la expansión de la matrícula secundaria fue sumamente significativa. Posteriormente, con el advenimiento del último gobierno dictatorial, se produjo un cese de dicha tendencia expansiva hasta entrados los años 80. Luego en el marco de la transición democrática, el Estado Nacional llevó adelante una serie de acciones que fueron desarmando la estructura autoritaria y propiciaron la democratización de la educación secundaria. Por ejemplo, la eliminación de las medidas selectivas dio lugar a una fuerte expansión de la matrícula y, en un contexto de escaso crecimiento económico, contribuyó a que comenzaran a plantearse, en el campo de la investigación educativa, nuevos análisis sobre la "masificación" (DE IBARROLA; GALLART, 1994; GALLART, 2006).

En síntesis, la evolución de la matrícula del nivel secundario se expande a lo largo de todo el siglo XX y principios del XXI. De acuerdo a la información estadística condensada en los documentos del Ministerio de Educación, hay un gran impulso a partir de la década del 50. Siempre constante, la evolución de la matrícula sólo fue interrumpida entre 1976 y 1983, es decir, durante el interregno dictatorial. Luego siguió en creciente expansión hasta 2002. (MINISTERIO DE EDUCACIÓN, 2007). ${ }^{5}$

\section{Situación educativa}

En Argentina el contexto de agudo deterioro laboral desde fines de los 90, que persistió por tres décadas, marchó en paralelo con una tendencia hacia la mayor escolarización de la población. Diversas investigaciones han destacado el aumento significativo del perfil educativo de la población en general, y de la fuerza de trabajo en particular, con especial relevancia entre los jóvenes. En base a los hallazgos de estudios anterio- 
res (MIRANDA; OTERO; CORICA, 2007), se ha podido observar que históricamente se verifica un importante incremento en el nivel educativo de los jóvenes comprendidos en la franja de 18 a 24 años de edad. El porcentaje de dicho grupo poblacional que obtuvo un título secundario mostró un incremento progresivo desde la década del 70 hasta inicios del siglo XXI, así como también ha ido en aumento - pero en mucho menor proporción - el número de quienes alcanzaron un diploma de nivel superior de instrucción. En este sentido, es en este grupo etario donde se evidencia el mayor aumento respecto de los niveles educativos alcanzados.

Con mayor precisión, en base a la información estadística del último relevamiento censal (INDEC-CENSO, 2001) sobre el total del grupo, alrededor de un tercio habían logrado finalizar con éxito el tramo de experiencia secundaria y un $24 \%$ se situaba en un nivel terciario o universitario incompleto. Como contracara, los porcentajes de aquellos que aún no habían finalizado el nivel de instrucción secundaria convivían con el $18 \%$ de quienes alcanzaron a completar sólo el nivel educativo primario y casi un $8 \%$ de aquellos que comenzaron, pero no finalizaron este primer nivel (Cuadro 1).

\section{CUADRO 1}

DISTRIBUICIÓN DE LOS JOVENES DE 18 A 24 AÑOS DE EDAD. SEGÚN SEXO Y MÁXIMO NIVEL EDUCATIVO ALCANZADO. TOTAL DEL PAIS. CENSO 2001

\begin{tabular}{c|c|c|c} 
& TOTAL $\%$ & VARONES \% & MUJERES \% \\
\hline Hasta primario incompleto & 7.9 & 9.0 & 6.8 \\
\hline Primario completo & 18.9 & 21.1 & 16.7 \\
\hline Secundario incompleto & 30.5 & 32.8 & 28.2 \\
\hline Secundario completo & 15.6 & 15.1 & 16.0 \\
\hline Terciario/Universitario completo & 24.9 & 20.7 & 29.2 \\
\hline Terciario/Universitario completo & 2.2 & 1.3 & 3.1
\end{tabular}

Considerando la distribución de los niveles educativos alcanzados para el grupo de 18 a 28 años de acuerdo al género, es en las mujeres entre quienes se registra la obtención de los más altos niveles de instrucción. Tal tendencia se manifiesta en los menores porcentajes de aquellas que obtuvieron sólo el nivel primario de instrucción formal y/o que han comenzado el ciclo medio sin finalizarlo aún. ${ }^{6}$ Como apuntan diversos estudios, esta tendencia en la Argentina ha ido en incremento en los registros censales de todo el siglo anterior. La propensión mayoritaria de la población femenina hacia el estudio no es una cuestión que se advierte 
sólo para este grupo etario, sino que marcha en sentido similar a lo registrado para la población argentina de 15 a 29 años de edad.

El avance en las tasas de escolarización del nivel secundario del último tiempo, impulsado en los periodos de democratización económica y política que favorecieron la fuerte incorporación de estudiantes, converge con una serie de cambios en materia de política educativa. Tales cambios adicionalmente actuaron en detrimento del carácter elitista citado como objetivo clave desde la misma creación del nivel medio.

En esta dirección, en las últimas tres décadas, se implementaron significativas modificaciones en la estructura normativa del sistema educativo argentino. Como ejemplo de ello, se pueden señalar las reformas establecidas por la Ley 24049 y la Ley $\mathrm{N}^{\circ} 24.195$, que avanzaron en la transferencia de los servicios educativos de nivel secundario y terciario a las provincias ${ }^{7}$. Parte de tal marco jurídico también estableció mutaciones en la estructura académica. La tradicional división entre el nivel primario (7 años) y secundario (5/6 años) sufrió alteraciones sustantivas. A partir de entonces, los primeros dos años del secundario pasaron a formar parte de un ciclo denominado Educación General Básica y los 4 últimos años a denominarse: Polimodal. Bajo este último se incluían una serie de modalidades agrupadas básicamente en cinco nomenclaturas: producción de bienes y servicios; economía y gestión de las organizaciones; humanidades y sociales; ciencias naturales y comunicación; y arte y diseño.

Ahora bien, durante el 2006 y luego de una revisión de la implementación del sistema polimodal, se procedió a un nuevo reajuste, más precisamente al establecimiento de un secundario compuesto por un ciclo básico común a todas las orientaciones y un ciclo diversificado, nuevamente con una duración total de 5 a 6 años. La sanción de la nueva Ley de Educación Nacional, $N^{\circ}$ 26.206, estableció estos lineamientos estructurales y además implementó la obligatoriedad de la escuela secundaria. El producto de las reformas puede condensarse en tres ejes medulares: avance de los procesos de descentralización institucional; modificación en la estructura de ofertas curriculares con nuevas opciones de orientación; y extensión de la obligatoriedad de la educación media. En su conjunto las medidas han modificando la fisonomía del sistema escolar argentino, implementaciones que se dan bajo el trasfondo de un agudo debate sobre las perspectivas de evolución, problematización del nivel medio y del papel que cumple la institución educativa en la sociedad. 
Los planteos no son novedosos, ya durante la década de los ochenta comenzaron a proliferar los diagnósticos acerca de la crisis del sistema educativo, en los cuales la situación de las escuelas secundarias cobró notoria difusión. El eje que vertebra las discusiones tiene que ver con "crisis y ausencia de sentido" de la educación de modo general y de la educación media en particular (TENTI FANFANI, 2003). Una serie de condiciones propiciaron la recarga de tintas sobre el nivel, la tendencia incremental de la cobertura ha dado lugar a la incorporación de sectores sociales antes excluidos de la propuesta, tensionando cada vez más su impronta selectiva, lo que configuró el marco de múltiples y nuevos desafíos para las instituciones escolares. Otro punto central, que comenzó a cobrar fuerza, fue el debate sobre la existencia de un sistema educativo integrado o, por el contrario, sumido en la lógica de lo fragmentario, con propuestas, experiencias y respuestas bien disímiles, frente a los problemas y de cara a la población estudiantil concurrente ${ }^{8}$.

En esencia, las opiniones críticas respecto del tramo secundario se orientaron sobre una articulación históricamente conflictiva: la relación entre educación y empleo. La renovación de la discusión entre las propuestas educativas y las demandas del mercado de trabajo estuvo en primer plano en los debates, pues la propuesta de movilidad ascendente con la que décadas atrás se asociara el pasaje por la educación media se veía severamente cuestionada ante la extensión del desempleo y las problemáticas laborales. A esto se sumaba el señalamiento de los fenómenos básicos de profundización de la segmentación y de crisis de identidad del nivel medio.

\section{Deserción y abandono escolar}

La masificación del nivel medio ha sido un fenómeno que marchó en paralelo con la persistencia del abano escolar. Complementando lo apuntado en el apartado anterior, de acuerdo al último Censo Nacional 2001, más de la mitad de los jóvenes de 20 a 29 años, el 52 porciento de dicha población, no había obtenido el titulo secundario. Además, siguiendo el documento del Ministerio de Educación (2008), no solo las tendencias regionales son variables respecto a la finalización del ciclo medio, sino que la terminalidad del nivel secundario sigue siendo un problema inquietante a principios del siglo XXI. En el acceso y la terminalidad del nivel 
superior se hacen evidentes también fuertes desigualdades sociales y regionales, generadas por la centralización de la oferta educativa en las grandes ciudades. Es decir que, apesar de los avances, en materia educativa se destacan problemáticas asociadas a la inclusión de educación secundaria y superior de los jóvenes de sectores sociales de menor capital económico y social'.

En este sentido, como han afirmado distintas fuentes bibliográficas del ámbito nacional, paralelamente al fuerte incremento registrado respecto del ciclo medio de educación formal, sigue coexistiendo un importante porcentaje de aquellos que no logran finalizarlo, es decir, que el abandono escolar es una temática vigente y de interés. En efecto, sigue existiendo una proporción de jóvenes que abandona el nivel, proporción que se ha mantenido relativamente constante en sucesivas generaciones: "Tal como ocurría varias décadas tras, hoy en día algo más de tres de cada diez ingresantes al nivel medio no llega a completarlo" (BISTONK; CERRUTI, 2005, p. 11).

Retomando el planteo de estas autoras para analizar en profundidad los determinantes del abandono escolar, debe partirse de entender que la decisión es una consecuencia de un proceso largo y complejo. A nivel del individuo es el resultado de la suma compleja de las experiencias individuales y familiares de cada joven, entre las cuales se incluyen aquellas relacionadas con factores de tipo socioeconómico, de socialización y contexto familiar.

Considerando los factores socioeconómicos, el nivel de ingresos del hogar resulta central, pues las dificultades económicas son por general antecedentes del ingreso del joven al mercado laboral. La participación laboral declina la posibilidad de dedicación exclusiva a actividades escolares. En muchas oportunidades la escasa compatibilidad entre el rol de estudiante y trabajador, contribuye a generar las condiciones propicias para el abandono escolar.

Asimismo, los logros educativos de los miembros de la familia son otro referente de influencia, en este punto la cadena trasmisora de posibilidades y expectativas favorables de las generaciones anteriores son un elemento considerable para la continuidad de los jóvenes en el sistema escolar, así como para la finalización exitosa. Las escasas experiencias educativas de las familias suelen no contribuir con la creación de un clima estimulante a la hora de permanecer en la escuela. 
Las actividades extraescolares que realizan los jóvenes y el contacto con su grupo de pares representan un núcleo más de factores que pueden actuar positiva o negativamente en la conducta respecto a la continuidad o deserción escolar. Una problemática preocupante, fundamentalmente entre las jóvenes, es la incidencia del embarazo adolescente que obstaculiza y suma circunstancias propensas para la irrupción de la cursada y el abandono escolar.

Si las actividades extraescolares y los grupos de pares representan un aspecto a tener en cuenta al momento de analizar la problemática, el contexto institucional en el cual se inscribe cada experiencia escolar conforma también un antecedente de peso. En este sentido, las prácticas pedagógicas y escolares propuestas y/o las características del ambiente escolar, entre otros elementos institucionales, pueden intervenir sustantivamente a la hora de la deserción. A ello se suma la influencia significativa de actores/autoridades escolares que actúen reforzando e incluso adelantando la salida de los "estudiantes problemáticos" (BISTONK; CERRUTI, 2005, p. 15).

El vínculo con los adultos y las relaciones inter-generacionales dadas al interior de las escuelas son un punto clave. Este tipo de relación cobra una presencia vital a la hora de analizar las diferencias entre jóvenes que, a pesar de estar expuestos a situaciones semejantes de contexto, logran alcances distintos en sus trayectorias escolares. Entre aquellos que logran finalizar con éxito el ciclo medio, la figura del adulto se ubica en un lugar central, representando un acompañamiento reconocido y valorado positivamente por los jóvenes. Se trata de un apuntalamiento positivo en tanto los adultos parecen ofrecer un "andamiaje" para el desarrollo de la propia subjetividad de los jóvenes frente a una sociedad cada vez más diversificada y compleja. En la misma dirección otros estudios han referido la importancia de los "adultos significativos" en el sostenimiento de las trayectorias juveniles no sólo en el ámbito de la educación, sino además en relación a otras dimensiones vitales (CACHON, 2000).

Ahora bien, es necesario comprender que el alcance de las problemáticas vinculadas a la falta de instrucción o a un escaso nivel educativo es múltiple. Circunscribiéndonos al aspecto laboral, por un lado, acarrean obstáculos a la hora de acceso al mercado laboral. Por otro, puede involucrar dificultades para el desempeño en la esfera laboral. Los jóvenes que no obtienen el título tienen, comparativamente, menos chances fren- 
te a las restringidas oportunidades de empleo. Investigaciones indican que para que los jóvenes puedan emplearse en sectores formales de la economía el título secundario es una calificación básica y las credenciales del nivel secundario son paradójicamente "cada vez más necesarias y cada vez más insuficientes” (FILMUS et. al., 2001).

Claro que el perjuicio que representa la falta de instrucción o los escasos logros educativos no se circunscribe al ámbito laboral, sino que abarca distintos aspectos cotidianos. Una experiencia escolar de mayor extensión acentúa las posibilidades de adquisición de una formación integral básica. En tanto que la combinación de dificultades que trae consigo, el abandono escolar puede atentar limitando posibilidades, ocasionando severas incidencias en el desarrollo de la autoestima y en las relaciones de los y las jóvenes con su entorno.

\section{Modalidad técnica}

Desde su surgimiento hasta nuestros días la modalidad técnica no estuvo exenta de vaivenes que comulgaron con el propio contexto, un proceso económico caracterizado por periodos de expansión y de crisis, en los cuales la industria ocupó un rol importante. En Argentina, en un primer periodo de expansión, la industria agrícola ganadera logró un importante desarrollo hasta fines del 1930. Por su parte, el modelo de sustitución de importaciones ha tenido su periodo de esplendor entre 1930 y 1960, pero ya desde los ochenta se comenzó a señalar la decadencia de la industria, acompasada y profundizada por las transformaciones del los años noventa con cambios jurídicos, organizacionales y tecnológicos de magnitud, procesos que impactaron tanto en el desarrollo como en la fisonomía de la escuela técnica.

La orientación técnica fue perdiendo protagonismo, reflejo de esta misma debacle. Al tiempo que, en su evolución interna fue sufriendo un creciente deterioro que implicó entre otros un retraso en los equipamientos acompañado de la escasa actualización de su especificidad tecnológica. Asociada a aquel primer periodo de esplendor, la modalidad generó una imagen social positiva y ligada a una relativa esperanza para los jóvenes de sectores populares que, en cierta forma, perdura hasta nuestros días. En términos generales, sobretodo antes de comenzar su etapa críti- 
ca, se conectó con las posibilidades más efectivas del aprendizaje de un oficio y una inserción laboral: un empleo en el campo industrial.

En el marco de la crisis general de la educación y en vistas a la profundidad de la crisis instalada en la modalidad técnica, surgieron nuevos planteos e intervenciones focalizados en superar su estancamiento y otorgarle el dinamismo perdido. Un saldo fundamental se materializó en la estipulación de una normativa específica: la Ley de Educación técnico Profesional $\mathrm{N}^{\circ} 26.058$ que, además de tener como objeto regular y ordenar la educación técnico profesional en el país, incluyó entre otros el establecimiento de fondos para diagnosticar el estado de situación y un presupuesto específico para contrarrestar la falta de infraestructura, etc.

En un reciente estudio exploratorio desarrollado durante el año 2006 se llevó a cabo un seguimiento de egresados de la educación técnico profesional en el territorio nacional. La intención de la investigación ha sido la creación de una base de datos y sistema de seguimientos, en miras al desarrollo de planes de mejora de las instituciones de educación técnico profesional en el marco del Registro Federal de Instituciones. El proyecto partió de tomar en cuenta un dato básico y actual: la existencia de 1.300 escuelas técnicas de nivel secundario en todo el país. Este conjunto abarca a establecimientos con distintas realidades institucionales, escuelas técnicas provinciales, polimodales con trayectos técnicos profesionales y ex escuelas nacionales (GALLART, 2006).

Sobre este conjunto de escuelas suscriptas en la propuesta de modalidad técnica el panorama es bien diverso. Las propuestas educativas de cada establecimiento, el equipamiento técnico, el perfil de los profesores con que cuenta, son solo algunas de las variables que establecen diferencias al interior del conjunto. Asimismo, enlazando la debacle del nivel técnico con el deterioro de la industria nacional y la profunda degradación de las relaciones salariales, la problemática del abandono de la modalidad técnica es un doble reto. Por un lado, es un hecho que desafía el esquema jurídico vigente en cuanto establece la obligatoriedad de la finalización del nivel secundario y consecuente en el formato técnico. Por otro, constituye un fenómeno que afecta con creces a jóvenes de los sectores más vulnerables de la población, sectores para los cuales, históricamente, la modalidad técnica se ha imaginado como posible apuesta y chance. 


\section{A modo de cierre}

A lo largo de este texto recorrimos brevemente el surgimiento y la inscripción de las escuelas medias en Argentina, así como también apuntamos las principales características de su matrícula y modificaciones en el marco jurídico que han transformado su estructura a lo largo de las últimas décadas. Posteriormente indagamos en el terreno de la problemática del abandono escolar, tomando en cuenta los principales factores de incidencia asociados a la deserción escolar, para ello, abordamos investigaciones recientes en el campo educativo. De las mismas se desprenden que el abandono escolar de la escuela media continúa siendo hoy una preocupación vigente en el panorama educativo argentino y, pese a que marcha en paralelo a la expansión de la matrícula escolar, su superación es un reto inexorable.

Las credenciales afectan múltiples aspectos de la dimensión de vida de los y las jóvenes y afectan sustantivamente las posibilidades de acceso a puestos de trabajo incidiendo a lo largo de las trayectorias laborales de los sujetos. En ese sentido, resulta pertinente seguir ahondando en la comprensión y análisis de la deserción escolar del nivel secundario y en particular ampliar nuestros conocimientos acerca de las especificidades de esta problemática en torno a la modalidad técnica. Esta modalidad, a la par de las oscilaciones de la industria y el contexto nacional, ha tenido un considerable momento de expansión, retraimiento y escasa modernización difícil de superar hasta nuestros días.

De acuerdo a las indagaciones realizadas en este campo, nos encontramos con un esquema de profundas diferencias en cuanto a la infraestructura y equipamientos al interior de las escuelas secundarias de una misma modalidad: técnica. De ello se desprende que, tanto la experiencia escolar como las posibles deserciones, están trazadas por los contextos institucionales y sus diferentes grados de desarrollo y evolución, al tiempo que estas diferencias son acentuadas entre los distintos territorios de la extensa Argentina.

La estrecha vinculación entre las posibilidades de inserción laboral posteriores al egreso de la escuela técnica son una imagen de profunda raigambre, dado el papel que en tiempos anteriores caracterizó el rol articulador de la modalidad con el desarrollo de un país con primacía en el desarrollo industrial. Junto con esas promesas diluidas y un esquema 
jurídico que avanza sobre la obligatoriedad de la finalización del secundario, es evidente la generación de un gran desafío pendiente. Ello fundamenta la necesidad de avanzar en la indagación sobre estas preocupantes situaciones combinadas: deterioro de la modalidad técnica y abandono escolar de la misma. 


\section{REFERÊNCIAS}

CACHÓN, L. Juventudes y empleos: perspectivas comparadas. Madrid: Editorial Injuve, 2000. 255 p.

BINSTOCK, G.; CERRUTTI, C. Carreras Truncadas: el abandono escolar en el nivel medio en la Argentina. Buenos Aires: UNICEF, 2005. p. 143.

DE IBARROLA, M.; GALLART, M.A. Democracia y productividad. Desafíos de una nueva educación media en América Latina. Santiago de Chile, Buenos Aires, México D.F.: OREAL (UNESCO) y CII, 1994.

DUSSEL, I. Curriculum, humanismo y democracia en la enseñanza media (1863/1920). Buenos Aires: FLACSO, 1997. 159 p.

FILMUS, D; KAPLAN, C; MIRANDA, A; MORAGUES, M. Cada vez más necesaria. cada vez más insuficiente, la escuela media en épocas de globalización. Buenos Aires: Editorial Santillana, 2001. p. 222.

GALLART, M. A. La escuela técnica industrial en Argentina: ¿un modelo para armar? Montevideo: CINTERFOR/OIT, 2006. 94 p.

GALLART, M. A. La construcción social de la escuela media. Buenos Aires: Editorial La Crujía Ediciones-Stella Edición, 2006. p. 172.

KESSLER G. La experiencia escolar fragmentada. Buenos Aires: IIPE-UNESCO, 2002. 116p.

MARSHALL, A. Empleo en la Argentina, 1991-1997: ¿Nuevas pautas de comportamiento después de la liberalización económica? Santiago-Chile: OIT, 1998. p. 25.

MINISTERIO DE EDUCACIÓN. LEY FEDERAL DE EDUCACIÓN, Nº 24.195 Disponível em: http://www.me.gov.ar/consejo/cf_leyfederal.html. Acesso em: 4 de setiembre 2009.

MINISTERIO DE EDUCACIÓN. LEY FEDERAL DE EDUCACIÓN, N 26.206. Disponível em: http://www.me.gov.ar/doc_pdf/ley_de_educ_nac.pdf. Acesso em: 30 de setiembre 2011.

MINISTERIO DE EDUCACIÓN. LEY DE EDUCACIÓN TÉCNICO PROFESIONAL, N² 26.058. Disponível em: http://www.me.gov.ar/doc_pdf/ley26058.pdf. Acesso em: 30 de setiembre 2011.

MINISTERIO DE EDUCACIÓN. La Obligatoriedad de la educación secundaria en Argentina. Deudas pendientes y nuevos desafíos. Buenos Aires: (DINECE) Dirección Nacional de Información y Evaluación de la Calidad Educativa, 2007.

MIRANDA, A; OTERO, A.; CÓRICA A. La educación secundaria y el mundo del trabajo en la Argentina poscrisis. Informe final eje Educación y Trabajo Investigación Proyecto: Intersecciones entre desigualdad y escuela media. Un análisis de las dinámicas de producción y reproducción de la desigualdad escolar y social en cuatro jurisdicciones, Dir. Inés Dussel, Agencia Secyt. (2005-2007), 2008.

MIRANDA, A.; OTERO, A.; CÓRICA, A. Cambio y situación social de los jóvenes en Argentina, In: Revista Papeles de Población, Centro de Investigación y Estudios Avanzados de la Población UAEM. Nueva Época, México, n. 52, abril-junio, 2007. p. 231-254.

OTERO, A. Jóvenes en la escuela media Argentina: opiniones en torno a las propuestas de formación para el trabajo en las distintas modalidades. In: Revista Question, Buenos Aires n. 19, invierno 2008. Disponível em:

http://www.perio.unlp.edu.ar/ojs/index.php/question/article/viewFile/643/553. 
Acesso em: 30 de setiembre 2009.

SVAMPA, M. A.; PEREYRA, S. Entre la ruta y el Barrio. 1. ed. Buenos Aires: Editorial Biblos, 2003. p. 230.

TEDESCO, J. C. Educación y sociedad en la Argentina 1880-1900. 2 ed. Buenos Aires: Centro Editor de América Latina, 1982. 169 p.

TENTI FANFANI, E. (comp.). Educación media para todos: Los desafíos de la democratización del acceso. Buenos Aires: Grupo Altamira, Unesco, Fundación OSDE, 2003. p.157.

TIRAMONTI, G. La trama de la desigualdad educativa: Mutaciones recientes en la escuela media 1. ed. Buenos Aires: Manantial, 2004. p. 245. 


\section{NOTAS}

1 Cabe aclarar aquí que más allá de esta imagen, ya desde la década del sesenta en la región latinoamericana se instalan discusiones de peso acerca del funcionamiento de la dinámica laboral. Se debatía sobre la expansión de formas atípicas de vinculación al mercado de trabajo que el capitalismo latinoamericano estaba generando, y sus consecuencias en las formas de integración social. Tanto la teoría de la marginalidad, como las discusiones sobre la informalidad, fueron expresiones del debate en organismos como el Programa Regional de Empleo para América Latina y el Caribe (PREALC).

2 La sociedad industrial fue portadora de una promesa integradora. En esa Argentina, el Estado se constituía como el eje organizador del conjunto social y la ética del trabajo era el principio fundante de la moral pública y privada. Fundidas en este esquema, la socialización familiar y escolar, y la disciplina fabril conformaban las instituciones soporte de ese orden industrial (TIRAMONATI, 2004).

3 Sobre todo tomando en cuenta, como lo hacen Svampa \& Pereyra, que cierta "excepcionalidad" de la Argentina en la región se atribuía al fuerte impulso de la sociedad salarial. Además, en comparación con otros países latinoamericanos, el país demostraba un escaso desarrollo de redes comunitarias por fuera del trabajo asalariado y una expansión inferior de trabajo informal como "actividad refugio" asociada tradicionalmente con las estrategias de supervivencia. (SVAMPA; PEREYRA, 2003, p. 12).

4 Esta autora señala que estas formas de intervención estaban fundamentadas en objetivos claves, como, entre otros, la eliminación de las prescripciones a la prerrogativa empresarial para contratar y despedir trabajadores; la reconstrucción del incentivo al trabajo; y la descentralización de la negociación colectiva. Entre marchas y contramarchas, las medidas puestas en funcionamiento no redundaron en la efectiva generación de empleos que formaba el corpus de sus objetivos iniciales.

5 En este sentido se argumentan y debaten la existencia de dos posibles hipótesis respecto al estancamiento a partir del 2002: 1. La escuela secundaria podría haber estado funcionando como un espacio refugio en momentos de retracción del mercado laboral; 2. La escuela secundaria estaría en estrecha competencia con la ampliación de posibilidades de inserción del mercado laboral a partir del 2002. Junto a ello se subraya la expansión matricular en las escuelas de adultos durante el periodo 2002/2006.

6 Respecto del nivel medio y siguiendo la información censal, el 16\% de las mujeres había obtenido el diploma secundario, porcentaje muy cercano al 15,1\% de los varones del grupo que también lo hicieron. La brecha diferencial se acrecienta en el nivel superior. El porcentaje de aquellas que lograron finalizar un ciclo superior Terciario/ Universitario alcanza el 3,1\%, mientras que entre los varones comprende sólo un 1,3\%. En una misma dirección, es en la población femenina entre quienes se observan los mayores porcentajes de Terciario/Universitario incompleto (29,2\% a 20,7\% de mujeres y varones, respectivamente). Resta señalar que, dada la extensión de las carreras universitarias del país, es dable esperar que se trate de grupos que van en camino a completar el nivel de formación superior.

7 Proceso que comienza a establecerse durante 1972. 
$8 \mathrm{Al}$ respecto, una investigación con jóvenes estudiantes del ámbito local en base a un trabajo empírico en instituciones secundarias de Buenos Aires ha señalado que se evidencia una profundización de "las experiencias de escolaridad fragmentada" (KESSLER, 2002, p. 24).

9 En este punto cabe aclarar que el abandono y la repitencia constituyen dos elementos asociados al fracaso escolar y al desarrollo de trayectorias educativas no-lineales. El término trayectorias no-lineales ha sido utilizado desde las investigaciones que han analizado de cerca la evolución de las tendencias educativas de la población y, en particular, de los/las jóvenes en edad teórica de asistencia a alguno de los ciclos educativos. Trayectorias no-lineales hace referencia: al ingreso tardío, a la repitencia y al abandono temporal, como episodios que generan trayectorias con sobresaltos, en donde se destaca la sobre-edad (la asistencia a niveles educativos que no se corresponden con la edad teórica prevista). El desarrollo de estos tipos de trayectorias educativas no-lineales alcanza mayor extensión entre los jóvenes de menores recursos económicos y sociales, siendo en el nivel medio donde se observa con mayor frecuencia la interrupción de la trayectoria escolar.

Recebido: $17 / 03 / 2010$

Aprovado: 20/10/2011

Contato:

Facultad Latinoamericana de Ciencias Sociales

Sede Académica Argentina Dirección Ayacucho 551, Capital Federal CP: C1026AAC

Buenos Aires

Argentina 